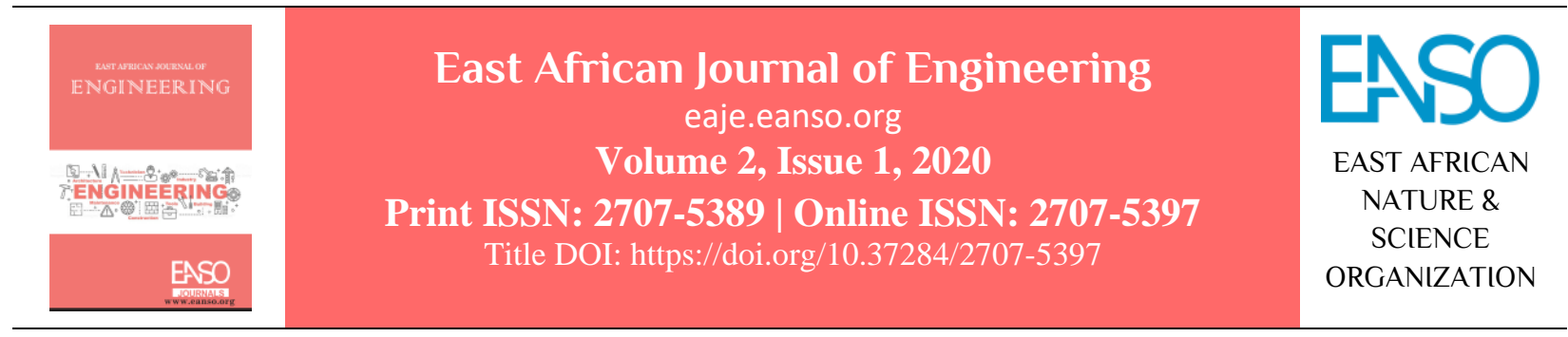

Original Article

\title{
The Suction Control Characteristics of Flow Separation on NACA 23012
}

\author{
Moses Oluwasina Julius ${ }^{1}$, Dr. Saheed Adewale Adio ${ }^{1 *}$, \\ Dr. Adam Olatunji Muritala ${ }^{1} \&$ Oluwasanmi Iyiola Alonge ${ }^{2}$ \\ ${ }^{1}$ Department of Mechanical Engineering, Obafemi Awolowo University, Ile-Ife, Osun State, Nigeria. \\ ${ }^{2}$ Department of Mechanical Engineering, Elizade University, Ilaramokin, Ondo State, Nigeria \\ *Author for correspondence Email: adiosa@ oauife.edu.ng; ORCID: https://orcid.org/0000-0002-2732-1360.
}

Article DOI: https://doi.org/10.37284/eaje.2.1.121

\section{Article history:}

Received: 27 Jan 2020

Accepted: 25 Feb 2020

Published: 10 Mar 2020

Keywords:

Flow Control,

Aerofoil,

Lift to Drag Ratio,

Suction and Boundary

Layer Separation

\section{ABSTRACT}

The enormous loss of momentum leads to stall and adversely affects the aerodynamic performance of aeroplane wings which may lead to a disaster, more importantly, risking the safety of the aeroplane by putting lives of passengers on it in danger. Therefore, this paper focuses on the enhancement of aerodynamic characteristics of NACA 23012 through the mitigation of flow separation and delay of the stall at higher angles of attack $\left(10^{\circ} \leq \alpha \geq 18^{\circ}\right)$ by using suction for Reynolds number $(R e)=3.4 \times 10^{6}$. Considering the different suction features such as suction width, suction position, and suction coefficient, the separation delay capability of a suction control is studied. Also, the lift to drag ratio and the impact of energy consumption variation during the control technique are used for estimating the control effects. The Reynolds Average Navier-Stokes (RANS) equations are employed together with the Menter's shear stress turbulent model. The result of this study revealed that the jet position just behind the separation point at $0.2 \%$ of the chord length shows an outstanding control outcome on the separation and stall, thereby increasing the lift. The lift to drag ration increased proportionately when the suction jet coefficient was increased. At suction coefficient $C_{q}=0.00225$, a $92.1 \%$ drag reduction and $72.7 \%$ lift enhancement is observed. Hence, the stall angle is moved beyond $21.5^{\circ}$ from an initial angle of $16^{\circ}$ and the more energy was saved at a high angle of attack.

\section{APA CITATION}

Julius, M., Adio, S., Muritala, A., \& Alonge, O. (2020). The Suction Control Characteristics of Flow Separation on NACA 23012. East African Journal of Engineering, 2(1), 1-13. https://doi.org/10.37284/eaje.2.1.121. 
East African Journal of Engineering, Volume 2, Issue 1, 2020

Article DOI: https://doi.org/10.37284/eaje.2.1.121

\section{CHICAGO CITATION}

Julius, Moses, Saheed Adio, Adam Muritala, and Oluwasanmi Alonge. 2020. "The Suction Control Characteristics of Flow Separation on NACA 23012”. East African Journal of Engineering 2 (1), 1-13. https://doi.org/10.37284/eaje.2.1.121.

HARVARD CITATION

Julius, M., Adio, S., Muritala, A. and Alonge, O. (2020) "The Suction Control Characteristics of Flow Separation on NACA 23012”, East African Journal of Engineering, 2(1), pp. 1-13. doi: 10.37284/eaje.2.1.121.

\section{IEEE CITATION}

M. Julius, S. Adio, A. Muritala, and O. Alonge, "The Suction Control Characteristics of Flow Separation on NACA 23012", EAJE, vol. 2, no. 1, pp. 1-13, Mar. 2020.

\section{MLA CITATION}

Julius, Moses, Saheed Adio, Adam Muritala, and Oluwasanmi Alonge. "The Suction Control Characteristics of Flow Separation on NACA 23012”. East African Journal of Engineering, Vol. 2, no. 1, Mar. 2020, pp. 1-13, doi:10.37284/eaje.2.1.121.

\section{INTRODUCTION}

The combined effect of adverse pressure gradient and skin friction occur more often at higher angle of attack or low Reynolds numbers, this induces enormous energy loss which results to the drop in the aerodynamic performances of aeroplanes during departure, landing and during manoeuvring of unmanned aerial vehicles (UAV) leading to large loss of momentum over the aerofoil. Therefore, many approaches have been deployed to stem the occurrence of these adverse situations in order to promote flow reattachment, thus enhancing the aerodynamic performance of the aircraft. Such methods could either be an active method which involves energy expenditure or passive method which does not involve energy spending. Over the past decades, suction control, which is an active technique has been one of the promising means which have been explored. Various scholars have considered different experimental and/or numerical technique to control flow on common NACA aerofoils in order to impede flow transition, enhance lift, postpone separation, reduce drag, suppress noise, and augment turbulence. Although Jacobs and Clay (1936) concluded that NACA 23012 has very good aerodynamic characteristics when compared to Clark $\mathrm{y}$ and other types of aerofoils, NACA 23013 is not one of the previously considered aerofoil using suction control technique.

Alrefai and Acharya (1996); Karim and Acharyat (1994) experimentally worked on the suction control of dynamic stall vortex of NACA 0012 at Reynolds number between $\mathrm{Re}=3.0 \times 10^{4}$ and $\operatorname{Re}=1.18 \times 10^{5}$ and a suction slot positioned between $2-5 \%$ of the chord length. There result showed complete mitigation of flow separation and dynamic stall over a well-defined area of parameter space. Owens and Perkins (1996) experimentally worked on the control of separation of the boundary layer on highly swept cranked delta wing via suction. Their results showed an increment in the lift to drag ratio to be $21 \%$ plus there was an effective enhancement of the aerodynamic characteristic of the wing. Wahidi and Bridges (2012) experimentally used suction techniques to restrain the size of the laminar separation bubble on LA2573a aerofoil. The suction technique effectively reduced the laminar bubble size and delayed the flow separation cum transition. They achieved a 14-24\% drag reduction and concluded that the consequence of using suction to reduce the drag is negligible. Chen et al. (2013) also experimentally worked on the suppression of vortex shedding on a cylinder using suction flow control technique. They concluded that the suction flow control on the circular cylinder diminished the alternate shedding of the vortex. Additionally, the instabilities in the coefficient of lift and drag of the cylinder were reduced drastically.

Atik and Walker (2005) investigated the impact of suction and suction/blowing as control mechanisms for separation that occur at the leading edge at high Reynolds number through a series of numerical simulation. They revealed that the suppression impact of a single suction control technique is better than the blow/suction control technique. Yousefi and Saleh (2015) and Yousefi et al. (2013a), (2014b) worked on the numerical optimization of suction parameters on the aerodynamic properties of NACA 0012. They concluded that suction 
having 0.5 amplitude and located at the leading edge between 1.75 and $12.5 \%$ of the chord length improved the aerodynamic properties of the aerofoil. That is, they observed the maximum lift, reduction in drag and stall improvement with these suction parameters. Carnarius et al. (2007) numerically worked on the steady flow over a NACA 4412 at Reynold number $\operatorname{Re}=10^{6}$. They revealed that application of steady suction at the upstream of separation point successfully controlled the flow separation and when the suction angle ranging from $\beta=20^{\circ}$ to $\beta=160^{\circ}$ was applied, it was found that suction perpendicular to the slot surface is optimal. Similarly, Azim et al. (2015) delayed boundary layer separation through suction on NACA 4412 and optimized the suction parameters. They got the suction location of 0.68 of the chord length and a suction pressure of $65 \mathrm{kPa}$ to be the most ideal for separation delay, consequently, the lift to drag ratio was improved by a factor of 2.24.

The preceding reviews have shown that suction located at an appropriate position can modify the distribution of pressure over an aerofoil surface as such produce a satisfactory effect on the coefficients of lift and drag; hence, mitigating the streamwise momentum loss that leads to the growth of the separation thickness. In the current study, the aerodynamic properties and performance of a NACA 23012 aerofoil, the impact of suction control and its parameters, and the impact of the energy absorbed during the process of control (using the figure of merit (FOM)) are numerically analysed at a Reynolds number of $3.4 \times 10^{6}$.

\section{NUMERICAL METHOD AND VALIDATIONS}

The two-dimensional fluid model was made a steady, chaotic, viscous and incompressible flow that has constant properties. The continuity and the momentum equations used in this study are the classical equations of fluid mechanics that governed the fluid dynamics as presented in Equations 1 and 2.

$\frac{\partial \overline{\mathrm{u}_{1}}}{\partial \mathrm{x}_{\mathrm{i}}}=0$

$\frac{\partial\left(\overline{\mathrm{u}_{1} \bar{u}_{\mathrm{j}}}\right)}{\partial \mathrm{x}_{\mathrm{j}}}=-\frac{1}{\rho} \frac{\partial \overline{\mathrm{P}}}{\partial \mathrm{x}_{\mathrm{i}}}+\frac{\partial}{\partial \mathrm{x}_{\mathrm{j}}}\left[\mathrm{v} \frac{\partial \overline{\mathrm{u}_{1}}}{\partial \mathrm{x}_{\mathrm{j}}}-\overline{\mathrm{u}_{\mathrm{i}}^{\prime} \mathrm{u}_{\mathrm{j}}^{\prime}}\right]$

where $\overline{\mathrm{u}_{1}^{\prime} \mathrm{u}_{\mathrm{j}}^{\prime}}$ integrates into the above equation of momentum the effects of turbulent instabilities (Alfonsi, 2009).

The turbulent model used to predict the mechanics of fluid and the behaviour of fluids around the aerofoil is Menter shear stress transport twoequation model $(\mathrm{k} \omega$-SST). The extrapolative capability of the k $\omega$-SST turbulent model is quite accurate for flow with separation. The calculation of boundary layer flow with separation is very much improved as a result of the turbulent model which includes $\mathrm{k}-\omega$ and $\mathrm{k}-\varepsilon$ standard models and the removal of the sensitivity of the $\mathrm{k}-\omega$ model to external flow. The Menter shear stress model is presented in Equations 3 and 4.

$\frac{\partial}{\partial \mathrm{x}_{\mathrm{i}}}\left(\rho \mathrm{U}_{\mathrm{i}} \mathrm{k}\right)=\widetilde{\mathrm{P}_{\mathrm{k}}}-\beta^{*} \rho \mathrm{k} \omega+\frac{\partial}{\partial \mathrm{x}_{\mathrm{i}}}\left[\left(\mu+\sigma_{\mathrm{k}} \mu_{\mathrm{t}}\right) \frac{\partial \mathrm{k}}{\partial \mathrm{x}_{\mathrm{i}}}\right]$

$\frac{\partial}{\partial x_{i}}\left(\rho U_{i} \omega\right)=\alpha \rho S^{2}-\beta \rho \omega^{2}+\frac{\partial}{\partial x_{i}}\left[\left(\mu+\sigma_{\omega} \mu_{t}\right) \frac{\partial \omega}{\partial x_{i}}\right]+2\left(1-F_{1}\right) \rho \sigma_{\omega 2} \frac{1}{\omega} \frac{\partial \mathrm{k}}{\partial x_{i}} \frac{\partial \omega}{\partial x_{i}}$

Where $\beta^{*}$ is 0.09 and $\sigma_{\omega 2}$ is 0.856 . To avoid the build-up of turbulence in the region of stagnation in the SST model calculations, a production limiter $\left(\mathrm{P}_{\mathrm{k}}\right)$ is used (Menter, 1992; Menter et al., 2003).

In this study, ANSYS Fluent is used for modelling and numerical analysis. The Reynolds number and the velocity of the free stream was $3.4 \times 10^{6}$ and $49.66 \mathrm{~ms}-1$ respectively. The geometry of NACA 23012 aerofoil, suction jet location, suction jet angle and the length of the suction jet are shown in detail in Figure 1. The length of the chord of the aerofoil is $1 \mathrm{~m}$; the length of suction jet for this investigation was $2.5 \%$ of the chord length (Yousefi et al., 2013), and the velocity of the suction jet was between 0 and 0.3 of the freestream velocity. The amplitude (A) is the ratio of the velocity of the jet to the velocity of the free stream. Therefore, the following three parameters which are suction 
coefficient $(\mathrm{Cq})$, dimensionless width of suction jet $\left(\mathrm{H}=\frac{\mathrm{h}}{\mathrm{C}}\right)$, suction jet location $\left(\mathrm{L}_{\mathrm{j}}\right)$ were investigated for optimum performance of the NACA 23012 aerofoil at high angles of attack. Since stall occurs on NACA 23012 without suction at around $16^{\circ}$ angle of attack (AOA), and flow separation begins around $10^{\circ}$, the above investigations were carried out between $0^{\circ}-18^{\circ}$ angles of attack. The components of the entrance velocity for the suction jet are defined as follows: $\mathrm{v}=\mathrm{u}_{\mathrm{jet}} \sin (\theta+\beta)$

$\mathrm{u}=\mathrm{u}_{\mathrm{jet}} \cos (\theta+\beta)$.

where $\beta$ is the angle between the direction of the velocity of the free stream and the surface of the local jet, and $\theta$ is the angle between the surface of the local jet and the direction of the velocity of the jet. The negative $\theta$ in Figure 1 symbolizes the condition for suction.

\section{Figure 1: Geometric Design of the Suction Mechanism on NACA 23012 and the Suction Jet} Parameters on the Aerofoil

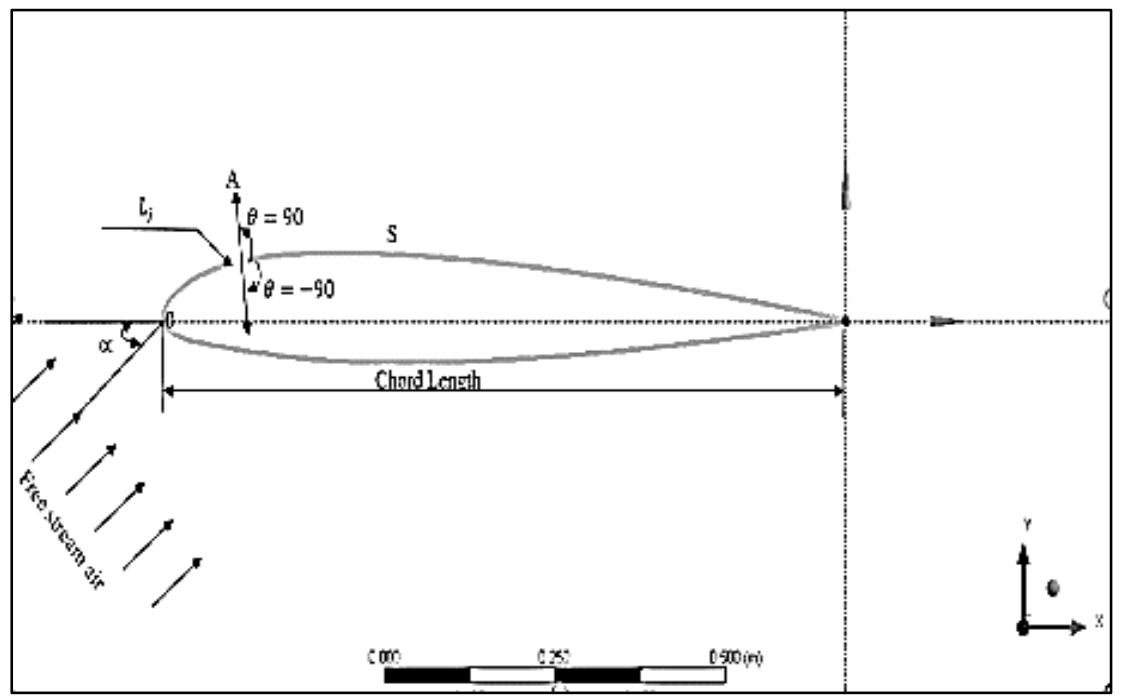

The equation governing the flow is discretize using the second-order upwind scheme. The systems of equations obtained during the discretization are solved through the procedure of Semi-Implicit Method for Pressure Linked Equations (SIMPLE) until all the dependent residual satisfy the convergence criterion of $O(5)$ reduction. The computational domain generated for simulation is a C-type structured grid which has multi-zone blocks as shown in Figure 2. In order to avoid the outer boundary of the computational domain from affecting the near flow field around the aerofoil, the area of the computational domain was made large. The value for velocity inlet is assigned to the inlet, and the lower and upper boundaries while the conditions for the outer boundary which is the freestream boundaries satisfy the Neumann condition. The boundary condition for the aerofoil surface is a no-slip, the turbulence intensity less than $0.15 \%$ for a low freestream is used which is a replica of the wind tunnel characteristics and the mesh having $\mathrm{y}+<1$ around the aerofoil is ensured.

The computations of the different sized grid are performed for NACA 23012 aerofoil at Reynolds number $3.4 \times 10^{6}$ and the test for grid independence is done on the calculated results through the study of coefficients of lift and drag. This is done at AOA of $10^{\circ}, 12^{\circ}$ and $16^{\circ}$ and for the fundamental case without the application of suction on the upper surface of the aerofoil. Figure 3 presented the test for grid dependency for the coefficient of lift and drag. From the Figure, the grid size with the fine and the better mesh was selected to be 758410 cells following the result of the grid independence that produced a reasonable accuracy. This mesh has a difference less than 0.01 from the preceding mesh as such the lift and drag ceased to have significant change as the number of elements increased. 
Figure 2: Structured Mesh of a NACA 23012 Aerofoil (a) meshing of the whole domain (b) boundary layer meshing around the aerofoil

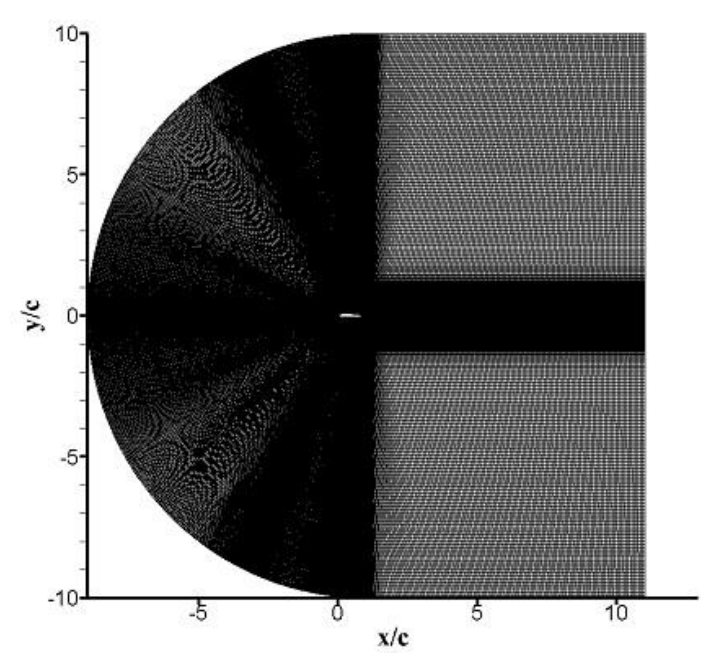

(a)

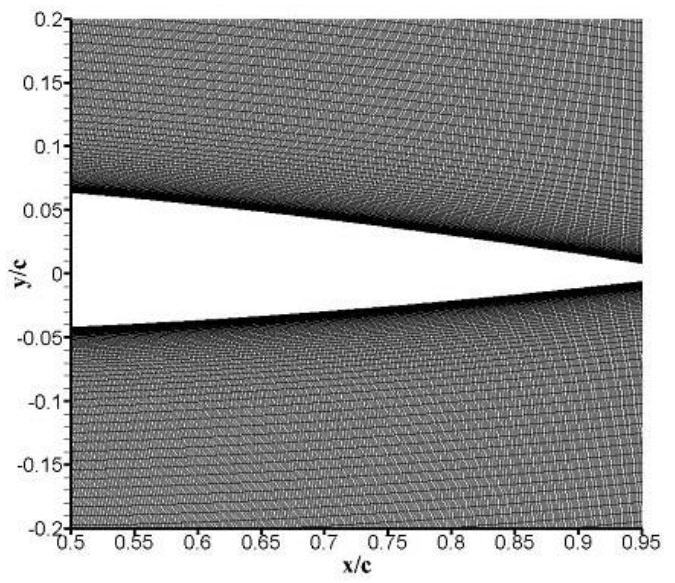

(b)

In order to validate the present numerical data, the coefficients of lift and drag are compared with the experimental values of Jacobs and Clay (1936), experimental values of Lee and Bragg (1999) and numerical values of Broeren et al. (2019). Jacobs and Clay (1936) worked on the characteristics of the NACA 23012 aerofoil under a Reynolds number of $3.4 \times 10^{6}$. Broeren et al. (2019) and Lee and Bragg (1999) did their investigation on NACA 23012 using Reynolds number of $4.6 \times 10^{6}$ and $1.8 \times 10^{6}$ respectively. The present work shows very good agreement with the two experimental data set (i.e. Jacobs and Clay (1936) and Lee and
Bragg (1999) as shown in Figure 4. The slight variations between the present data and the experimental data could be as a result of some uncertainties such as the type of turbulence model used here, pitching aerofoil vs static aerofoil used, etc. The accuracy of lift to drag ratio and the prediction of stall depends on the type of turbulent model selected for simulation. The k- $\omega$ ST model has better stall prediction capability. To the best of the authors' knowledge, the exact experimental data for suction are not available in the open literature.

Figure 3: Mesh independence study (a) Lift Coefficient (b) Drag Coefficient.

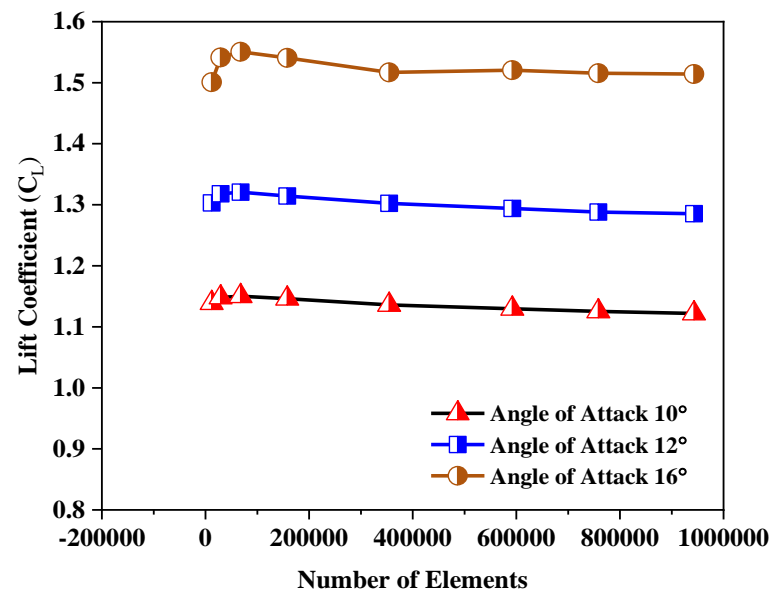

(a)

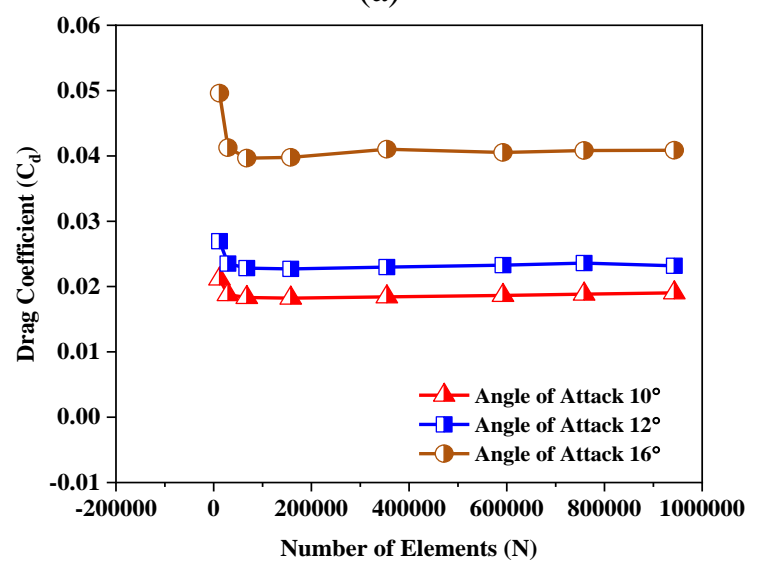

(b) 
Figure 4: Comparison between lift coefficient of present numerical work, numerical and experimental results

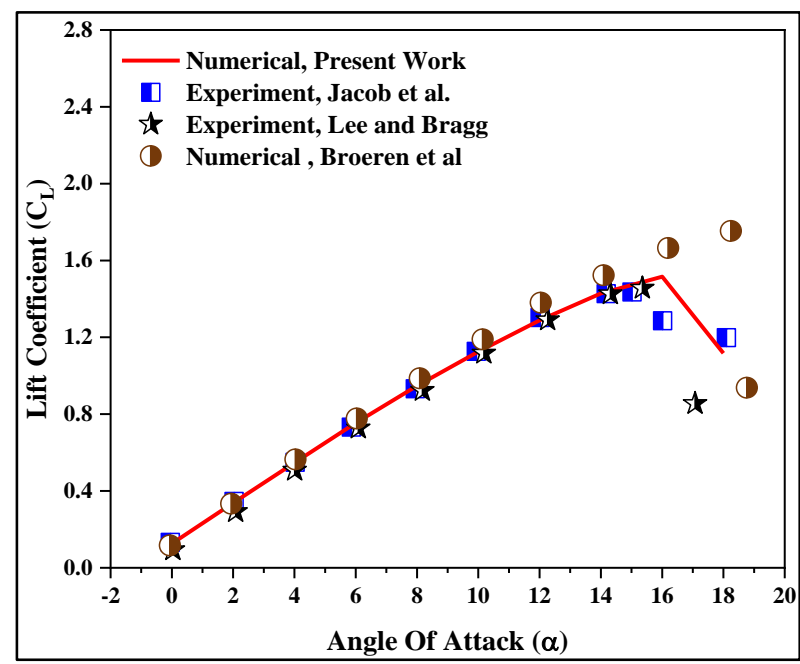

\section{Simulating the Suction Mechanism}

The suction amplitude and suction coefficient are used to quantify the control energy consumption as expressed in Yousefi et al. (2014).

$\mathrm{C}_{\mathrm{q}}=\frac{\mathrm{Q}}{\rho \times \mathrm{C} \times \mathrm{u}_{\infty}^{2}}=\frac{\rho \times \mathrm{h} \times \mathrm{u}_{\mathrm{j}}^{2}}{\rho \times \mathrm{C} \times \mathrm{u}_{\infty}^{2}}=\frac{\mathrm{h}}{\mathrm{C}} \times \frac{\mathrm{u}_{\mathrm{j}}^{2}}{\mathrm{u}_{\infty}^{2}}$

$\mathrm{H}=\frac{\mathrm{h}}{\mathrm{C}}$

$\mathrm{C}_{\mathrm{q}}=\mathrm{H} \times \mathrm{A}^{2}$

The mesh structures of the suction slot are shown in Figure 5. The first slot is located at $0.05 \mathrm{c}(0.5 \%$ of cord length) and varied between $0.05 \mathrm{c}$ to $0.7 \mathrm{c}$ layers of fluid within the boundary layers with low momentum. The means fluid that has lost its kinetic energy and has the tendency to lead to flow separation and later cause the shedding of the vortex at the upper surface of the aerofoil is absorbed through suction to maintain steady flow around the aerofoil.
Figure 5: Mesh Distribution around the Suction Slot

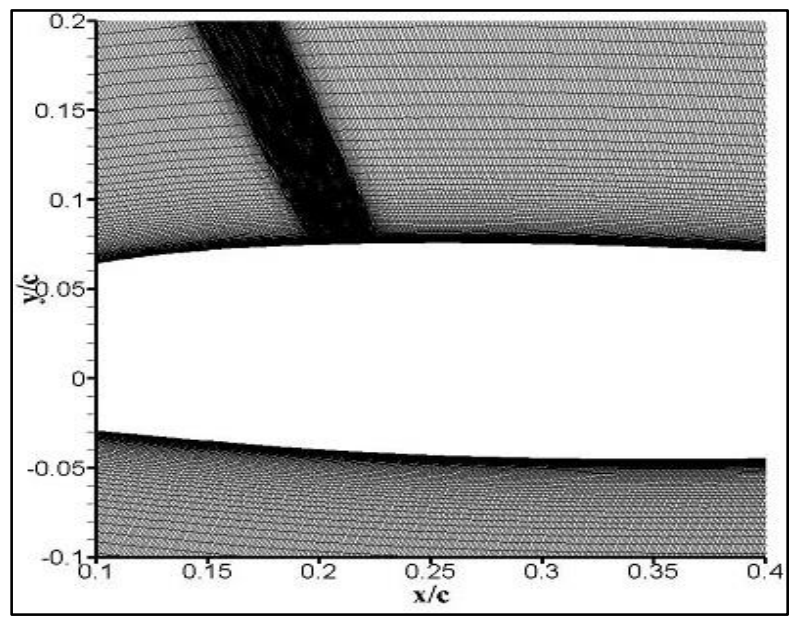

Figure 6: Flow characteristic and velocity distribution at a different angle of attack. (a) lift coefficient (b) velocity distribution at $8^{\circ} \mathrm{AOA}$ (c) velocity distribution at $16^{\circ} \mathrm{AOA}$ (d) velocity distribution at $18^{\circ} \mathrm{AOA}$.



(a)

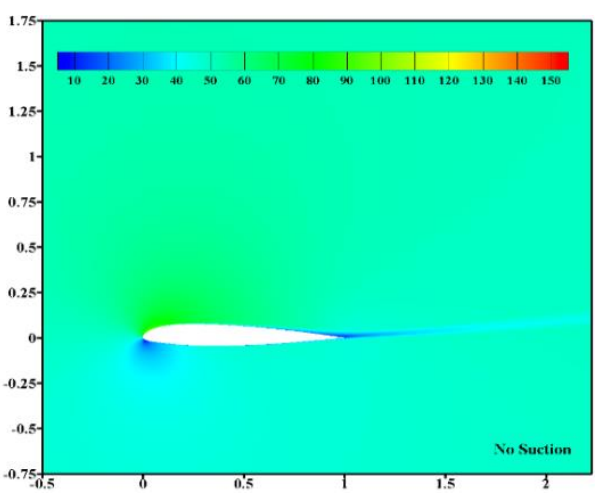

(b) 


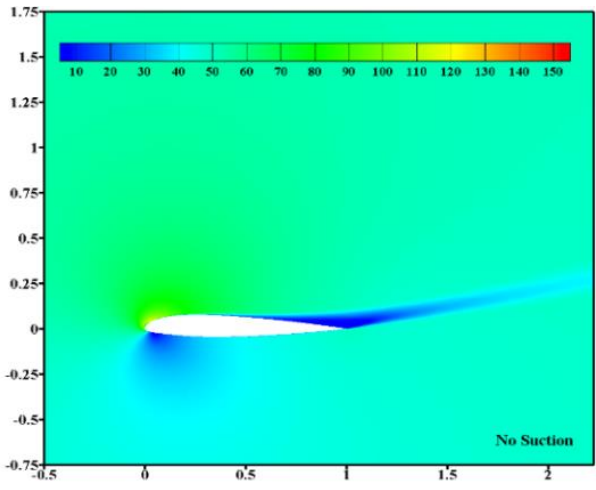

(c)

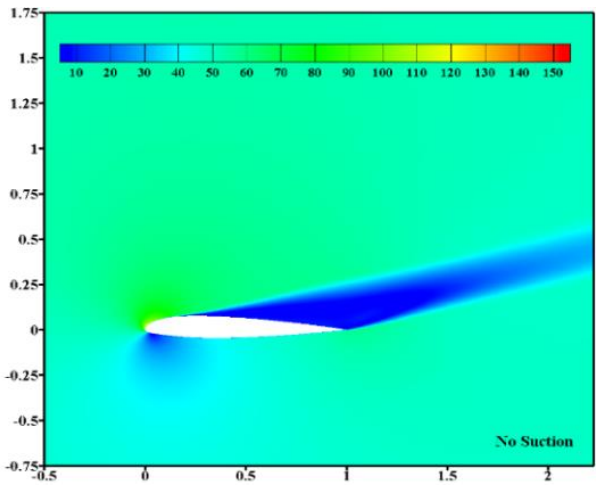

(d)

\section{RESULTS AND DISCUSSIONS}

Figure 6 shows the lift characteristic and velocity distributions around the aerofoil at three different angles of attack. It shows clearly that at the lower angle of attack ranging from $0^{\circ}$ to $\sim 10^{\circ}$, there was no separation and there is a full attachment of flow around the aerofoil. Therefore, this explains the linearity of the lift coefficient curve at angles of attack that are below $10^{\circ}$. At the angle of attack beyond $10^{\circ}$, flow separation begins, non-linearity of lift curve begins and lift to drag ratio declines due to the influence of adverse pressure gradient until stall at 16 degrees where there is a drop in the lift.

\section{Suction Parameter Effects on Flow Control}

The impact of suction on the flow field around aerofoil is dependent on the suction parameters which includes the suction coefficient, suction location, suction width etc. Because suction can delay or suppress flow separation at the expense of energy cost, it is necessary to study the cost of energy and suppression effect as a combination of the different suction parameters. This will have different cost implications in relation to energy consumption and the different suppression effects.

\section{Suction Slot Location}

In enhancing the aerodynamic performance of suction by mitigating the flow separation at constant $\mathrm{C}_{\mathrm{q}}$, it is thereby necessary to place the suction in the appropriate position. The dependency of suction position on a various parameter such as Reynolds number, amplitude, angle of attack (AOA), suction coefficient etc. makes it very complex to choose the best suction location because these parameters affect the suction location. Therefore, to make the complex decision easy, the performance of the aerofoil is measured at different locations on the suction side of the aerofoil at suction coefficient $0.00025(\mathrm{~A}=0.1)$, Reynolds number of $3.4 \times 10^{6}$ and AOA of $10 \mathrm{o}, 14 \mathrm{o}$ and 18o. Figure 7 shows the variation in the lift to drag ratio at a different suction location. According to the velocity contour shown in Figure 8 , the separation point at the leading edge region of the aerofoil is about $0.3 \mathrm{c}$ for aerofoil without suction at AOA $18^{\circ}$.

When a suction slot is situated at a region very close to the leading edge i.e. suction slot at $0.2 \mathrm{c}$, the point of separation is moved downstream of the flow towards the trailing edge (i.e. at about 0.90c from the leading edge of the aerofoil). Contrarily, if the location of suction is too close to the leading edge such as $0.05 \mathrm{c}$ or the location of suction is towards the region of the trailing edge such as $0.4 \mathrm{c}, 0.5 \mathrm{c}$, $0.7 \mathrm{c}$ etc. this drastically deteriorates the aerodynamic performance of the aerofoil. This explains that moving the location of slot for suction away from $0.2 \mathrm{c}$, either way, will produce a catastrophic separation and the aerodynamic performance drops as such the increase in turbulence causes increase in drag coefficient and decrease in the lift when compared to aerofoil without suction, hence, there is a drastic fall in the lift to drag ratio. To have a better performance of the aerofoil, the slot for suction is done at $0.2 \mathrm{c}$. For instance, an angle of attack $14^{\circ}$, slot location $0.2 \mathrm{c}$ decreases the coefficient of drag by $44.4 \%$ and the lift to drag ratio increases by $78.3 \%$ when compared to aerofoil that has no suction. 
Figure 7: Comparison between Lift to Drag Ratio and Suction Location

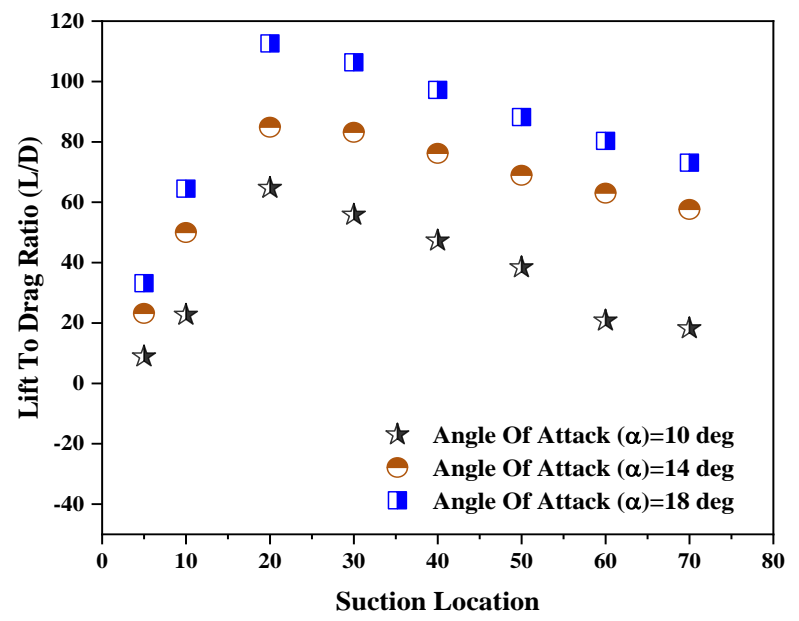

Figure 8: Velocity contour for $\mathrm{AOA}=18$ degrees (a) not suction; (b) suction slot at $0.2 \mathrm{c}$ of the aerofoil, and (c) with a suction slot at $0.7 \mathrm{c}$ of the aerofoil

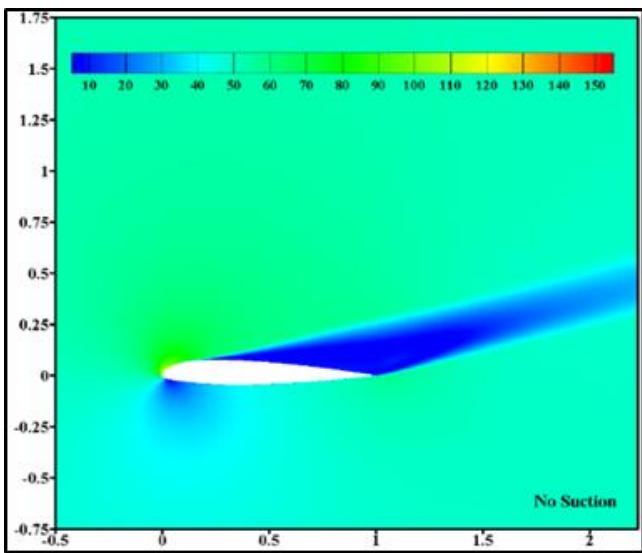

(a)

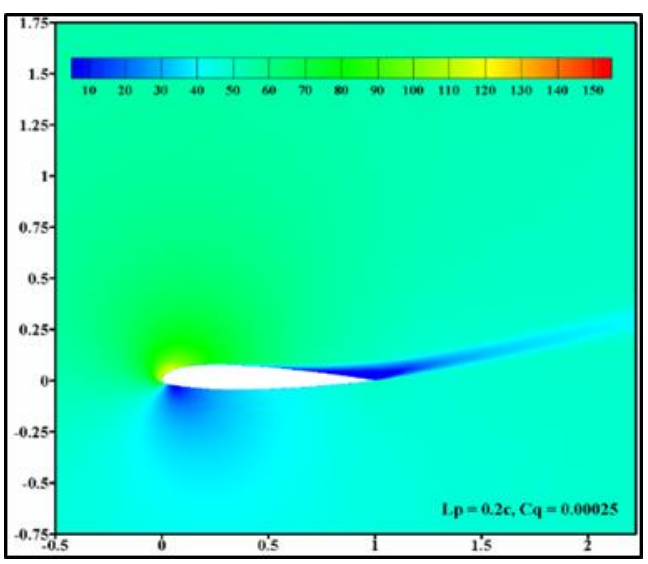

(b)

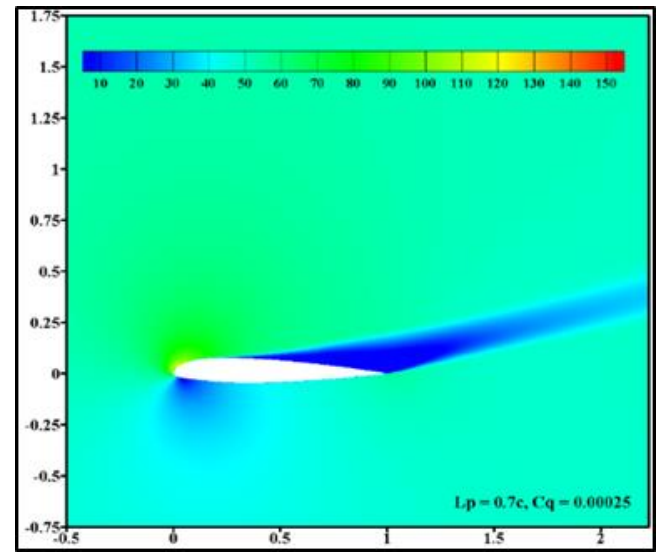

(c)

\section{Suction Width}

Figure 9 illustrates the effects of different sizes of the suction slot, at the optimized suction location, on the coefficient of lift. The suction coefficient used is 0.00225 and it can be seen that at a lower angle of attack the variation in the lift with respect to changes in the width of suction is almost negligible. However, there are significant changes in the lift with respect to changes in the width of suction as the angle of attack increases. At higher angles of attack, there is a continuous increase in the lift coefficient as the width of suction increases until a suction width of $2.5 \%$ of the chord length when the lift value plateaued. For instance, at an angle of attack 160 , there is a $2.2 \%$ increase in the lift coefficient as the width of the suction is varied from $1.5 \%$ to $2.5 \%$ of the chord length. A further increase to $3.0 \%$ of the chord length only produced $0.48 \%$ increase in the lift coefficient. Therefore, the suction width that optimized the aerodynamic performance of the aerofoil at the leading edge is $2.5 \%$ of the chord length. 
Figure 9: The Changes in The Lift Coefficient with Different Suction Width at Suction Coefficient of 0.00225 And Angle of Attack 10 ${ }^{\circ}$, $12^{\circ}, 14^{\circ}$ and $16^{\circ}$



\section{Suction Coefficients}

The point of separation of the fluid shifted to the region of the trailing edge of the aerofoil as the coefficient of suction increase from 0.00025 to 0.00225 . The initial introduction of the suction slot with the suction coefficient of 0.00025 on the aerofoil shifted the point of separation to the vicinity of the trailing edge but when the suction coefficient is increased, there is a huge improvement in the position of the separation point as shown in Figure 10. For instance, at $\mathrm{AOA}=18^{\circ}$, separation position moves from about $0.2 \mathrm{c}$ to about $0.526 \mathrm{c}$ of the aerofoil when the suction coefficient of 0.00025 was introduced on the aerofoil. This is further moved downstream of the flow to $0.9 \mathrm{c}$ when the suction coefficient is increased to 0.00225 . Suction coefficient of 0.00225 not only make the flow field on the upper surface steady but also delay separation which could have led to a stall.
Figure 10: The Changes in Separation Point with Increase in Suction Coefficient at Angle of Attack $1^{\circ}, 1^{\circ}$, And $18^{\circ}$

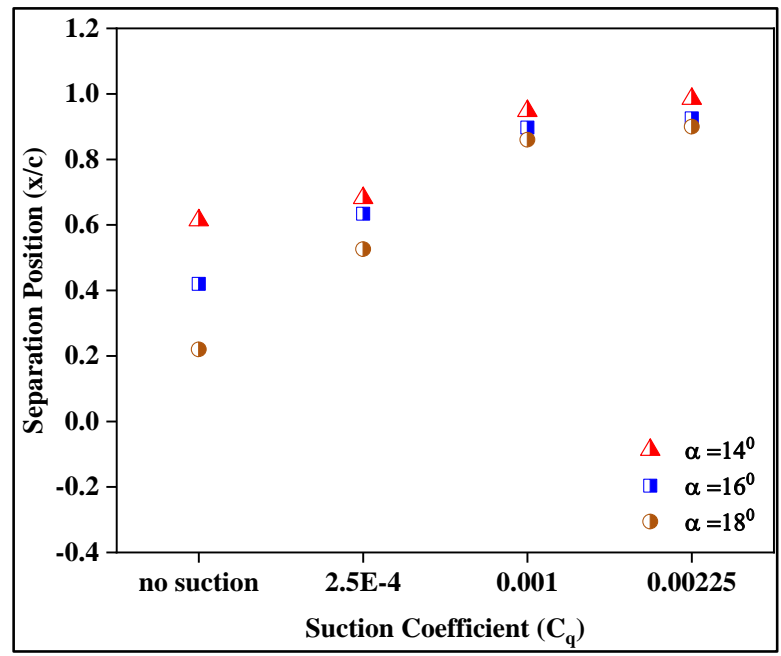

\section{Flow Control Mechanism with a Single Suction Slot}

The optimisation of flow control through suction around aerofoil is dependent on the suction parameter used. The suction width is considered to be $2.5 \% \mathrm{c}$ and the distance from the leading is $0.2 \mathrm{c}$. Figure 11 shows the velocity contour for flow pass aerofoil with different suction coefficients $\left(\mathrm{C}_{\mathrm{q}}\right)$ at $\operatorname{Re}=3.4 \times 10^{6}$ and $\propto=18^{\circ}$. The point at which the separation of flow occurs shifted towards the trailing edge as the suction coefficient is increased. The stall is delayed at $\mathrm{C}_{\mathrm{q}}=0.00225$ and the flow field structure becomes steady. Figure 12 shows the variations in lift coefficient (CL), drag coefficient $(\mathrm{Cd})$, and the lift to drag ratio (L/D) with respect to the suction coefficient. It should be noted that as the suction coefficient increases the drag coefficient decreases. Here, the sum of the coefficient of friction drag and the coefficient of pressure drag is equal to the total drag coefficient (Schlichting and Gersten, 2017), however, the pressure drag is dominant in the present study. The decrease in the drag coefficient is due to the large reduction in the pressure drag coefficient gradient. An increase in the coefficient of suction automatically induces an increase in the coefficient of lift and this is possible only when the enormous energy loss is recovered through the suppression of flow separation (i.e. suction is applied to absorb layers of flow that have lost its momentum). Since the drag reduced and lift 
is enhanced, the lift to drag ratio is also enhanced, which is a positive result. Therefore, with the suction control of $\mathrm{C}_{\mathrm{q}}=0.00225$, there is an improvement in the aerodynamic properties of the aerofoil; the coefficient of lift increase by about $72.7 \%$ and the coefficient of drag decrease by about $92.1 \%$ while the stall angle is moved to about $21.5^{\circ}$ at $\mathrm{AOA}=18^{\circ}$.

Figure 11: The velocity contours with different suction coefficients $\left(C_{q}\right)$ at $\mathrm{AOA}$ of $18^{\circ}$ (a) no suction (b) $\mathrm{A}=0.1$ at, $C_{q}=0.00025$, (c) $\mathrm{A}=0.3$ at, $C_{q}=0.00225$

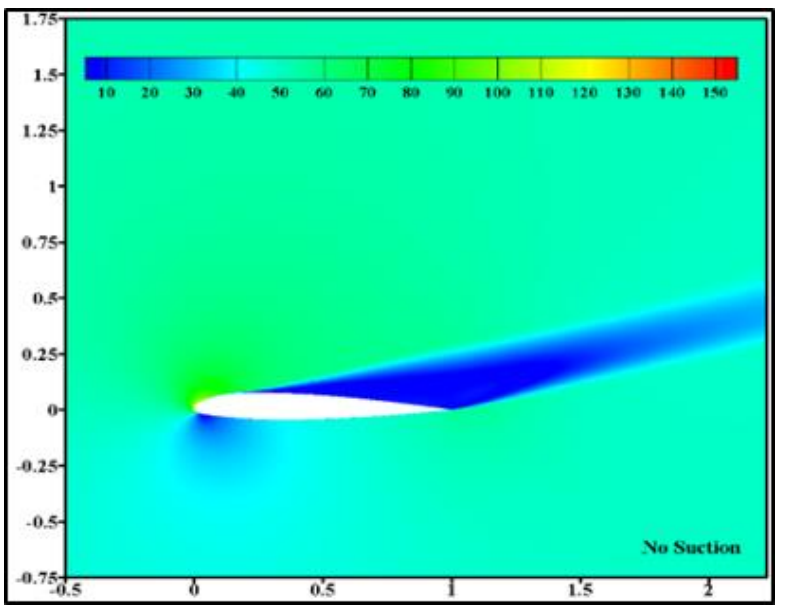

(a)

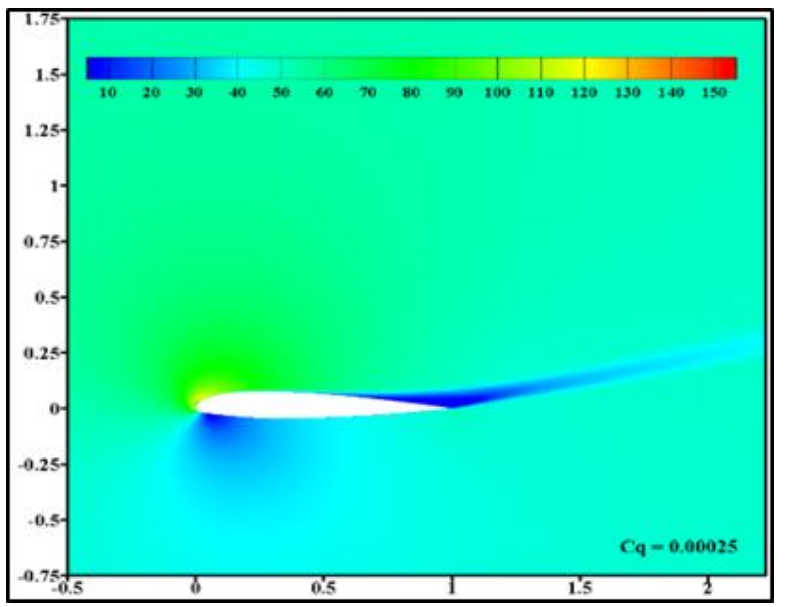

(b)

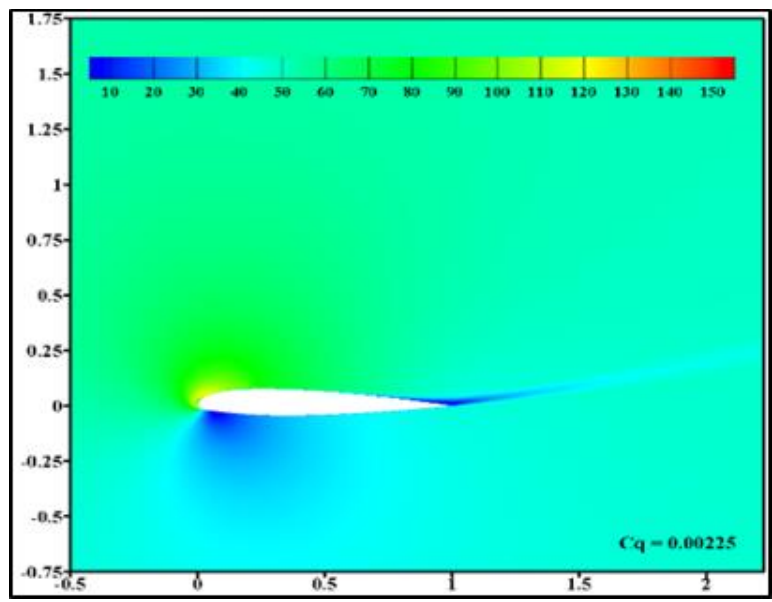

(c)

Figure 12: The variation in the aerodynamic properties at various angles of attack (a) lift coefficient, $C_{L}$, (b) drag coefficient, $C_{d}$, (c) lift to drag ratio, $L / D$, with respect to the suction coefficient.

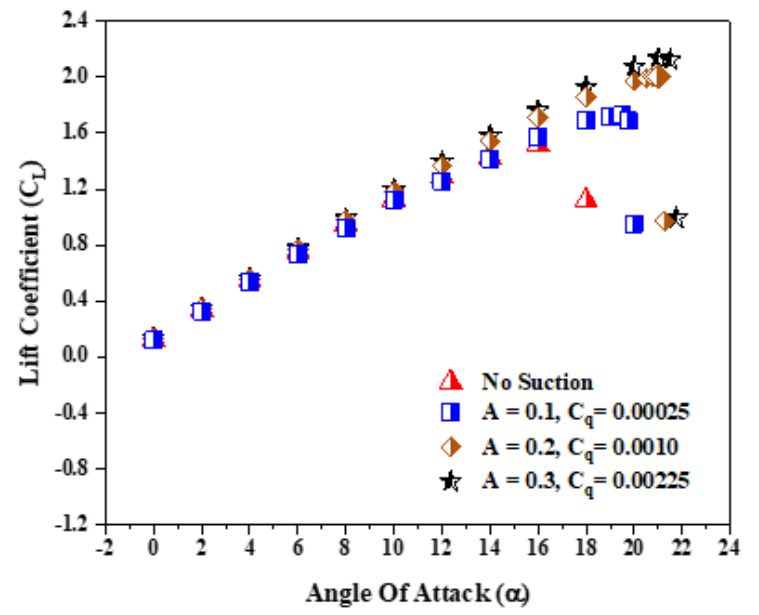

(a)

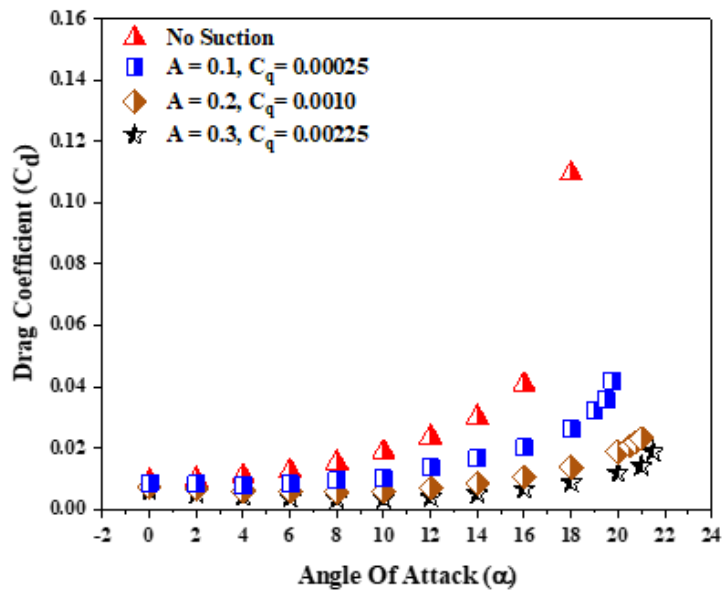

(b) 


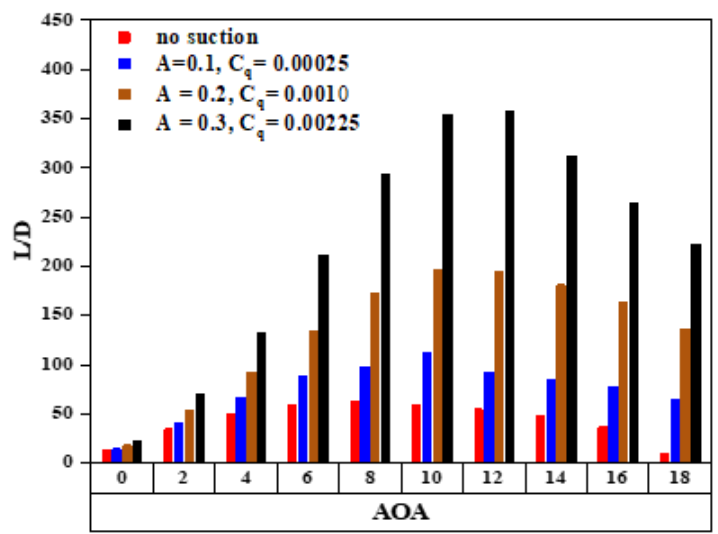

(c)

Figure 13 shows the coefficient of the pressure of the suction control at the suction coefficient $\mathrm{C}_{\mathrm{q}}=$ 0.00225 compared with the coefficient of the pressure of the baseline (aerofoil without suction). For both conditions, the distribution of pressure on the upper surface and the lower surface of the aerofoil is negative and positive respectively. The application of suction control on the upper surface modifies the distribution of pressure around the aerofoil. The pressure at the leading edge of the aerofoil rapidly decreases and also towards the trailing edge region of the aerofoil the pressure increases; thereby, improving the capability of the aerofoil to resist the adverse pressure gradient and separation of flow from occurring. Therefore, the introduction of suction control results in an increase in lift through pressure reduction on the entire upper surface and increment in pressure on the entire lower surface.

Figure 13: The comparison of the pressure coefficient of the suction control at the suction coefficient $C_{q}=0.00225$ at $\mathrm{AOA}=16^{\circ}$ with no suction



\section{The Impact of Absorption Energy of Suction Flow Control}

From Figure 12 it is evident that as the suction coefficient increases, it results in the increase in the lift to drag ratio and this shows that a lot of energy could be saved through the application of suction control, in other words, enormous energy loss due to flow separation is regained. Also, suction as active flow control method can consume energy, therefore, it is not possible to unconditionally improve the aerodynamic properties of an aerofoil. So, it is necessary to evaluate the cost of energy used to absorb the layers of fluid that loss its momentum, and the gain obtained in drag reduction and lift enhancement. The estimation of the energy cost of suction is done by assuming that it is equivalent to the required power to cause chamber static pressure to be equal to the static pressure of the mainstream. and it is presented mathematically in Equation (10) (Zhang et al., 2017).

$\mathrm{P}_{\text {suction }}=\left(\mathrm{P}_{\infty}-\mathrm{P}_{c}\right) \mathrm{Q} /\left(\rho \mathrm{U}_{\infty}\right)$....

The power in relation to the equivalent suction drag $\mathrm{D}_{\mathrm{s}}$ is expressed in Equation (11)

$\mathrm{P}_{\text {suction }}=\mathrm{D}_{\mathrm{s}} \times \mathrm{U}_{\infty}$

Hence, the coefficient of the equivalent suction drag can be expressed in Equation (12)

$C_{d_{-} s}=\frac{D_{s}}{\frac{1}{2} \rho_{\infty} U_{\infty}^{2} \mathrm{c}}=\frac{\left(\mathrm{P}_{\infty}-\mathrm{P}_{\mathrm{c}}\right)}{\frac{1}{2} \rho_{\infty} \mathrm{U}_{\infty}^{2}} \cdot \frac{\mathrm{Q}}{\rho \mathrm{U}_{\infty}^{2} \mathrm{c}}=\frac{\left(\mathrm{P}_{\infty}-\mathrm{P}_{\mathrm{c}}\right)}{\frac{1}{2} \rho_{\infty} \mathrm{U}_{\infty}^{2}} \mathrm{C}_{\mathrm{q}}$

Where $\mathrm{C}_{\mathrm{q}}$ is established in equation 7,8 , and 9 . The figure of merit (FOM) is used for both the drag $\left(\mathrm{FOM}_{\mathrm{cd}}\right)$ and the lift $\left(\mathrm{FOM}_{\mathrm{cl}}\right)$ to evaluate the benefit of using the suction for drag reduction and lift enhancement. It is expressed mathematically in Equations (13) and (14).

$\mathrm{FOM}_{\mathrm{cd}}=\frac{\mathrm{C}_{\text {baseline }}-\mathrm{C}_{\mathrm{d}_{\text {suction }}}}{\mathrm{C}_{\mathrm{d}_{\_} \mathrm{s}}}$

$\mathrm{FOM}_{\mathrm{cl}}=$

$\frac{\mathrm{C}_{\mathrm{l} \_ \text {baseline }}-\mathrm{C}_{\text {l_suction }}}{\mathrm{C}_{\mathrm{d} \_s}}$

When the FOM $>1$, it indicates that it requires a low cost of energy to reducing a large amount of drag or producing an enhancement in the lift. The higher the value of FOM the better the performance 
improvement of the aerofoil. Figure 14 presents the variation of FOM with respect to the suction coefficient, $\mathrm{C}_{\mathrm{q}}=0.00225$. It is obvious that both the $\mathrm{FOM}_{\mathrm{cd}}$ and $\mathrm{FOM}_{\mathrm{cl}}$ values are greater than 1 which indicates that the suppression of the boundary layer saves energy by drag reduction within this area at the suction coefficient. Also, it is evident that at a low angle of attack less or negligible amount of energy was saved, but at a higher angle of attack, an enormous amount of energy was saved. In relation to Figure 11 and Figure 12, it is at this suction coefficient that the separation of flow is completely suppressed, and the maximum lift to drag ratio is attained. Therefore, the suction control with suction coefficient, $\mathrm{C}_{\mathrm{q}}=0.00225$ has a reasonably high lift, low drag and overall saves energy.

Figure 14: The distribution of FOM against the angle of attack (a) lift coefficient (b) drag coefficient
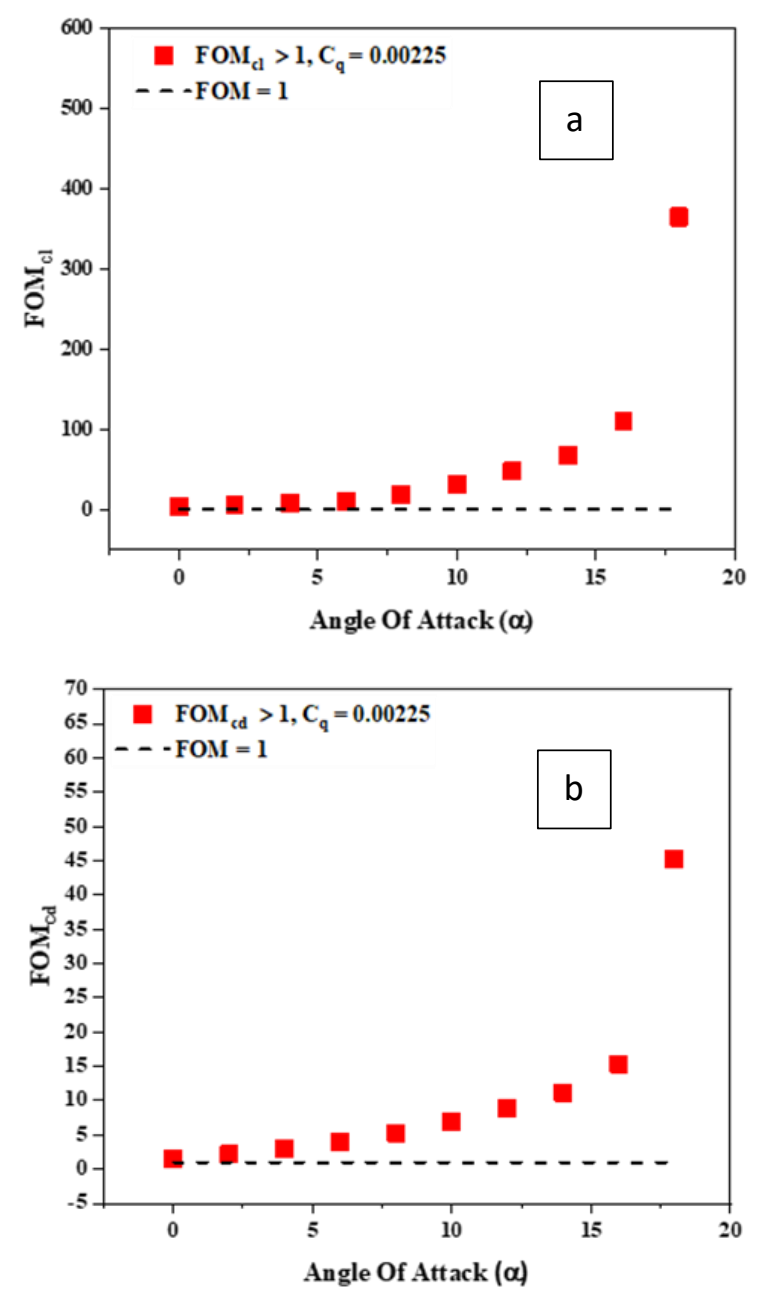

\section{CONCLUSION}

The ANSYS Fluent was used to study the characteristics of the flow past a NACA 23012 aerofoil at $\operatorname{Re}=3.4 \times 10^{6}$, angle of attack up to $18^{\circ}$. The results from the present investigation were validated by comparing with the data from previous experimental and numerical investigations. At Reynolds number $\operatorname{Re}=3.4 \times 10^{6}$, the angle of the stall, $\propto_{\text {stall }}=16^{0}$ and beyond was used as a study case to discuss the effect of using suction as a control technique in this investigation. The detached flow past an aerofoil was reattached when suction as a control technique was introduced on the suction side of the aerofoil. The point of separation of the flow was shifted to the vicinity of the trailing edge, the stall was delayed and the instability of the boundary layer was made stable as the coefficient of suction $\mathrm{C}_{\mathrm{q}}$, was increased. With the variation of suction width with respect to lift coefficient, the optimum suction width was obtained to be at $2.5 \%$ of the chord length. At the optimised suction coefficient, the FOM showed that there was energy saving as relatively small suction power was able to restore the momentum loss at high AOA.

\section{REFERENCES}

Alfonsi, G. (2009). Reynolds-averaged NavierStokes equations for turbulence modelling. Applied Mechanics Reviews, 62(4).

Alrefai, M. D., \& Acharya, M. (1996). Controlled leading-edge suction for management of unsteady separation over pitching airfoils. AIAA Journal, 34(11), 2327-2336.

Atik, H., \& Walker, D. (2005). Boundary-layer Separation Control Using Local Suction and Injection. In 4th AIAA Theoretical Fluid Mechanics Meeting (p. 4937).

Azim, R., Hasan, M. M., \& Ali, M. (2015). Numerical investigation on the delay of boundary layer separation by suction for NACA 4412. Procedia Engineering, 105, 329334.

Broeren, A. P., Addy Jr, H. E., Bragg, M. B., Busch, G. T., \& Montreuil, E. (2011). Aerodynamic simulation of ice accretion on airfoils. 
Carnarius, A., Gunther, B., Thiele, F., Wachsmuth, D., Tröltzsch, F., \& de los Reyes, J. C. (2007). Numerical study of the optimization of separation control. In 45th AIAA Aerospace Sciences Meeting and Exhibit (p. 58).

Chen, W., Liu, Y., Xu, F., Li, H., \& Hu, H. (2014). Suppression of vortex shedding from a circular cylinder by using a travelling wave wall. In 52nd Aerospace sciences meeting (p. 0399).

Jacobs, E. N., \& Clay, W. C. (1936). Characteristics of the NACA 23012 airfoil from tests in the full-scale and variable-density tunnels. Langley Field.

Karim, M. A., \& Acharya, M. (1994). Suppression of dynamic-stall vortices over pitching airfoils by leading-edge suction. AIAA Journal, 32(8), 1647-1655.

Lee, S., \& Bragg, M. B. (1999). Experimental investigation of simulated large-droplet ice shapes on airfoil aerodynamics. Journal of Aircraft, 36(5), 844-850.

Menter, F. R., Kuntz, M., \& Langtry, R. (2003). Ten years of industrial experience with the SST turbulence model. Turbulence, heat and mass transfer, 4(1), 625-632.

Menter, F. (1992). Improved two-equation k- $\omega$ turbulence models for aerodynamic flows NASA Ames, CA. NASA Technical Memorandum, 103975.

Owens, D., \& Perkins, J. (1996, January). Improved performance on highly swept wings by suction boundary-layer control. In 34th Aerospace Sciences Meeting and Exhibit (p. 431).

Schlichting, H., \& Gersten, K. (2017). Boundarylayer theory. Springer.

Wahidi, R., \& Bridges, D. H. (2012). Effects of distributed suction on an airfoil at low Reynolds number. AIAA Journal, 50(3), 523539.

Yousefi, K., \& Saleh, R. (2015). Three-dimensional suction flow control and suction jet length optimization of NACA 0012 wing. Meccanica, 50(6), 1481-1494.

Yousefi, K., Saleh, R., \& Zahedi, P. (2014). Numerical study of blowing and suction slot geometry optimization on NACA 0012 airfoil. Journal of Mechanical Science and Technology, 28(4), 1297-1310.

Yousefi, K., Saleh, S. R., \& Zahedi, P. (2013). Numerical investigation of suction and length of suction jet on aerodynamic characteristics of the NACA 0012 airfoil. International Journal of Materials, Mechanics and Manufacturing, 1(2), 136-142.

Zhang, W., Zhang, Z., Chen, Z., \& Tang, Q. (2017). Main characteristics of suction control of flow separation of an airfoil at low Reynolds numbers. European Journal of MechanicsB/Fluids, 65, 88-97.

\section{NOMENCLATURE}

$\begin{array}{ll}\alpha & \text { aerofoil angle of attack [degree] } \\ \alpha_{\text {stall }} & \text { angle of attack at stall } \\ \mathrm{c} & \text { aerofoil chord length [m] } \\ C_{q} & \text { suction coefficient [-] } \\ \mathrm{H} & \text { dimensionless jet width [-] } \\ C_{d} & \text { drag coefficient [-] } \\ C_{L} & \text { lift coefficient [-] } \\ \text { AOA } & \text { the angle of attack [degree] } \\ U_{\text {ave }} & \text { average velocity [m/s] } \\ P_{c} & \text { local static pressure of the slot [kPa] } \\ C_{d_{-} \text {baseline }} & \text { drag coefficient without suction [-] }\end{array}$

$\mathrm{X} / \mathrm{c}$
$\mathrm{Re}$
$L_{j}$
$\mathrm{~A}$
$L_{p}$
$\rho$
$\mathrm{N}$
$\bar{P}$
$P_{\text {suction }}$
$D_{\text {s }}$
$C_{d_{-} \text {suction }}$

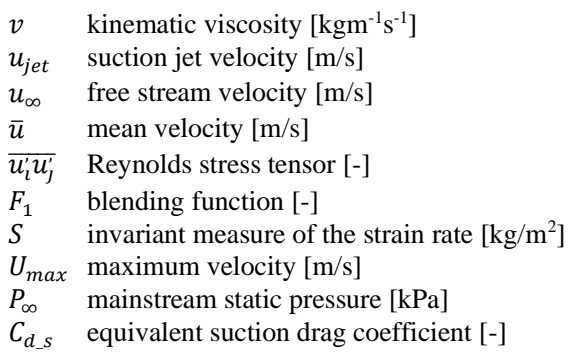

